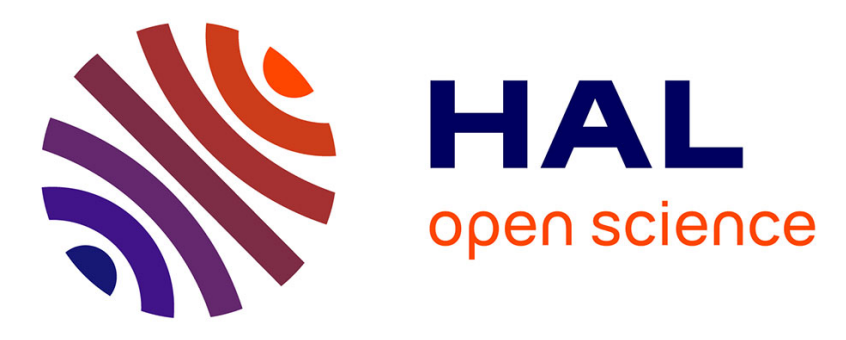

\title{
Safe- and eco-driving control for connected and automated electric vehicles using analytical state-constrained optimal solution.
}

Jihun Han, Antonio Sciarretta, Luis Leon Ojeda, Giovanni de Nunzio, Thibault Laurent

\section{To cite this version:}

Jihun Han, Antonio Sciarretta, Luis Leon Ojeda, Giovanni de Nunzio, Thibault Laurent. Safeand eco-driving control for connected and automated electric vehicles using analytical stateconstrained optimal solution.. IEEE Transactions on Intelligent Vehicles, 2018, 3 (2), pp.163-172. 10.1109/TIV.2018.2804162 . hal-01990552

\section{HAL Id: hal-01990552 \\ https://hal-ifp.archives-ouvertes.fr/hal-01990552}

Submitted on 13 Feb 2019

HAL is a multi-disciplinary open access archive for the deposit and dissemination of scientific research documents, whether they are published or not. The documents may come from teaching and research institutions in France or abroad, or from public or private research centers.
L'archive ouverte pluridisciplinaire HAL, est destinée au dépôt et à la diffusion de documents scientifiques de niveau recherche, publiés ou non, émanant des établissements d'enseignement et de recherche français ou étrangers, des laboratoires publics ou privés. 


\title{
Safe- and eco-driving control for connected and automated electric vehicles using analytical state-constrained optimal solution
}

\author{
Jihun Han, Antonio Sciarretta, Luis Leon Ojeda, Giovanni De Nunzio, Laurent Thibault
}

\begin{abstract}
Speed advisory systems have been proposed for connected vehicles in order to minimize energy consumption over a planned route. However, for their practical diffusion, these systems must adequately take into account the presence of preceding vehicles. In this paper, a safe- and eco-driving control system is proposed for connected and automated vehicles to accelerate or decelerate optimally while guaranteeing vehicle safety constraints. We define minimum inter-vehicle distance and maximum road speed limit as state constraints, and formulate an optimal control problem minimizing the energy consumption. Then, an analytical state-constrained solution is derived for realtime use. A feasible range of terminal conditions is established, and such conditions are adjusted to guarantee the existence of the analytical solution. The proposed system is evaluated through simulation for various driving scenarios of the preceding vehicle. Results show that it can significantly reduce energy consumption and also avoid collision without increasing trip time. Moreover, the proposed system can serve as an energy-efficient advanced cruise control by setting a short prediction horizon.
\end{abstract}

Index Terms-Connected and automated vehicles, Electric vehicles, Speed advisory system, Adaptive cruise control, Ecodriving control, Optimal control.

\section{INTRODUCTION}

$\mathbf{T}$ HANKS to the development of communication technologies (e.g. vehicle-to-vehicle, vehicle-to-infrastructure, etc.) fused with on-board sensors (e.g. radar, lidar, vision camera, etc.) and global navigation systems, vehicles have been equipped with connectivity and automation technologies over the past years. Connected and automated vehicles (CAVs) have easier access to the required traffic information, therefore they can be controlled more precisely compared to humandriven vehicles. With these benefits, CAVs can reduce the number of traffic accidents caused by human error and improve traffic flow stability and throughput.

One of the automated functions, adaptive cruise control (ACC), aims to track a desired speed while maintaining a prescribed inter-vehicle distance. For heavy-duty vehicles, the ACC can reduce inter-vehicle distance, thereby decreasing energy consumption due to the decrease in aerodynamic drag resistance. However, this energy saving benefit may not be achieved for personal CAVs because the ACC may

J. Han was with Department of Control, Signal and System, IFP Energies Nouvelles, Rueil Malmaison, France in 2016-2017 and is currently with Oak Ridge National Laboratory, Oak Ridge, USA (e-mail: hanj1@ornl.gov).

A. Sciarretta, L. Leon Ojeda, G. De Nunzio, and L. Thibault are with Department of Control, Signal and System, IFP Energies Nouvelles, RueilMalmaison, France (e-mail: antonio.sciarretta@ifp.fr; luis.leon-ojeda@ifp.fr; giovanni.de-nunzio@ifp.fr; laurent.thibault@ifp.fr). result in sub-optimal speed profiles with aggressive acceleration/deceleration [1]. For this reason, optimization-based ecodriving functions, which try to maximize energy efficiency, have been recently added to ACC in CAVs.

In these energy-efficient ACC approaches, the conflicting objectives of speed/distance tracking and energy efficiency are merged in a single cost function to be minimized in model predictive control (MPC) framework. MPC relies on a prediction horizon over which an linear/nonlinear optimization is performed [2], [3]. Nonlinear optimization methods outperform linear ones, but they increase computational time [4], [5]. Although fast numerical algorithms based on Pontryagin's minimum principle have been recently proposed, they still limit the prediction horizon that can be used in real-time [6], [7], [8], [9].

Eco-driving techniques can also be employed for speed advisory systems (SAS), which primarily define energy consumption as a cost function to be minimized, allowing a larger speed control range than in ACC [10]. In [11], [12], [13], dynamic programming is used to compute optimal speed trajectories as a reference for the driver, but this algorithm is not suitable for real-time applications due to its high computational time. In contrast, a few studies such as [14], [15], [16] have attempted to derive and use closed-form optimal speed trajectories. However, in these contributions, vehicle safety constraints imposed by neighboring vehicles are not considered. In other studies, such constraints are simplified by coordinating trip times of all CAVs for special driving scenarios, such as highway on-ramps [17] or urban traffic intersections [18], [19].

The previous studies focusing on the SAS do not explore the possibility of directly taking into account the presence of the preceding vehicle as a state constraint. Thus, as an extension to the ACC (vehicle safety) and the SAS (energy efficiency), this paper is intended to investigate how to derive a closed-form state-constrained optimal solution and to propose a robust and computationally efficient MPC that ensures the existence of an analytical solution. The optimization problem will be formulated with the objective of minimizing the energy expenditure of a CAV driving in a traffic stream, while avoiding collisions and respecting the road speed limits.

The paper is organized as follows: Section 2 is a brief summary of the safe- and eco-driving control problem. In Section 3, derivation of the state-constrained solution is described. Section 4 presents the feasible conditions for the stateconstrained optimal solution. In Section 5, several case studies 
are analyzed through simulation, and the results are discussed. Finally, in Section 6, the conclusions drawn from this study are presented.

\section{SAFE- AND ECO-Driving Control Problem}

\section{A. System Model}

To capture vehicle's motion, as well as its energy efficiency including the powertrain loss, a basic longitudinal model is used:

$$
\begin{aligned}
\dot{s} & =v, \\
m \dot{v} & =F_{t}-\left(F_{a}+F_{r}+F_{g}\right)-F_{b}, \\
& =F_{t}-\rho_{a} c_{d} A_{f} v^{2} / 2-c_{r} m g-m g \sin (\alpha(s))-F_{b},
\end{aligned}
$$

where $F_{t}, F_{a}, F_{r}, F_{g}$, and $F_{b}$ are the traction force at the wheels, the aerodynamic drag resistance, the rolling resistance, the hill climbing resistance, and the mechanical brake force, respectively; $s$ and $v$ indicate the vehicle's position and speed, respectively; $m$ is the vehicle mass, $\rho_{a}$ is the external air density, $A_{f}$ is the vehicle frontal area, $c_{d}$ is the aerodynamic drag coefficient, $c_{r}$ is the rolling resistance coefficient, $g$ is the gravity acceleration, and $\alpha$ is the road slope as a function of the position.

An electric vehicle is propelled by an electric motor connected to a transmission. The traction force through the transmission is described under the assumption of no slip at the wheels, as follows:

$$
F_{t}=\left(T_{m} \eta_{t}^{\operatorname{sign}\left(T_{m}\right)} R_{t}\right) / r
$$

where $T_{m}$ is the motor torque, $R_{t}$ is the transmission ratio, $\eta_{t}$ is the transmission efficiency, and $r$ is the wheel radius.

Electric power consumed by the electric motor is usually modeled as a tabulated function of the motor torque and speed resulting from steady-state experimental data (motor map). However, to derive the analytical optimal solution for online implementation, the approximated closed-form expression [20] is used,

$$
P_{m}=V_{a} i_{a}=\omega_{m} T_{m}+\left(R_{a} / k^{2}\right) T_{m}^{2}=b_{1} v T_{m}+b_{2} T_{m}^{2},
$$

where $b_{1}:=R_{t} / r, b_{2}:=r_{a} / k^{2}, V_{a}, i_{a}$, and $R_{a}$ are some effective voltage, current, and resistance, respectively, and $k$ is the motor torque constant. Note that $V_{a}=i_{a} R_{a}+k \omega_{m}$, $i_{a}=T_{m} / k$, and the rotational motor speed is $\omega_{m}=R_{t} v / r$.

If the electrochemical power drained from or supplied to the battery system is $P_{b}$, then the energy consumption of an electric vehicle is computed by $E_{f}=\int_{0}^{t_{f}} P_{b} d t$. In eco-driving studies for electric vehicles, the electrochemical conversion efficiency in the battery can be simplified to a constant value or neglected [10]. Here, $P_{b}$ is set to $P_{m}$.

\section{B. Problem Statement}

The main goal of the controller is to minimize the energy consumption of the host electric vehicle, defined by (4), while guaranteeing the vehicle safety. In this respect, a first state inequality constraint is set by the requirement that the vehicle speed cannot exceed the maximum speed limit $\left(v_{\max }\right)$,

$$
h_{1}(t)=v(t)-v_{\max } \leq 0,
$$

and a second constraint is set by the requirement that the intervehicle distance is always larger than a minimum safe distance $\left(\delta_{s}\right)$,

$$
h_{2}(t)=s(t)-\left(s_{p}(t)-\delta_{s}\right) \leq 0,
$$

where $s_{p}$ is the position of the preceding vehicle.

The control inputs, $T_{m}$ and $F_{b}$, are bounded as

$$
\begin{aligned}
T_{m . \min } & \leq T_{m}(t) \leq T_{m \cdot \max } \\
F_{b . \min } & \leq F_{b}(t) \leq 0 .
\end{aligned}
$$

\section{Model Predictive Control Problem Formulation}

A model predictive control (MPC) approach is used to solve the safe- and eco-driving control problem in real-time. At every time step, the MPC computes an optimal control trajectory over a finite prediction horizon $\left(t_{p}\right)$, and this process with feedback of current vehicle information is repeated as the prediction horizon recedes. If the control inputs are defined by $u:=T_{m}$ and $W:=F_{b} / m$, the MPC problem is formulated using (1-8):

$$
\begin{aligned}
\operatorname{minimize} & J=\int_{t_{0}}^{t_{0}+t_{p}}\left(b_{1} v u+b_{2} u^{2}\right) d t \\
\text { subject to } & \dot{s}=v, \\
& \dot{v}=c_{1} \eta_{t}^{\operatorname{sign}(u)} u-\left(c_{2} v^{2}+c_{0}\right)-W, \\
& u_{\min } \leq u(t) \leq u_{\max } \\
& W_{\min } \leq W(t) \leq 0 \\
& h_{1}(t)=v(t)-v_{\max } \leq 0 \\
& h_{2}(t)=s(t)-\left(s_{p}(t)-\delta_{s}\right) \leq 0
\end{aligned}
$$

where $c_{1}:=R_{t} /(r m), c_{2}:=\rho_{a} A_{f} c_{d} /(2 m)$, and $c_{0}:=g\left(c_{r}+\right.$ $\sin (\alpha(s)))$, while $t_{0}$ is the current time.

Initial and terminal state constraints are

$$
\begin{aligned}
& s\left(t_{0}\right)=s_{0}, \quad v\left(t_{0}\right)=v_{0}, \\
& s\left(t_{0}+t_{p}\right)=S, \quad v\left(t_{0}+t_{p}\right)=V,
\end{aligned}
$$

where $S$ is a desired terminal position, $s_{0}$ is the current position, $v_{0}$ is the current speed, and $V$ is a desired terminal speed of the receding horizon.

The MPC solver is important to determine the real-time implementability of the controller. Many studies have developed efficient numerical algorithms, but they are still computationally expensive, thereby limiting the prediction horizon that can be used in real-time [21], [22]. In this paper, rather than using numerical algorithms, state-contrained optimal solutions are analytically derived under some assumptions, and used as the solution to (9)-(14) at each time $t_{0}$.

\section{Analytical State-CONSTRained Solution}

For simplicity, the receding prediction horizon is expressed as $\left[0, t_{p}\right]$ instead of $\left[t_{0}, t_{0}+t_{p}\right]$. To derive the analytical solution, further assumptions are required: 1) no transmission loss $\left.\left(\eta_{t}=1\right), 2\right)$ no mechanical brake force $\left.(W=0), 3\right)$ no control input constraints $\left(u_{\max }=-u_{\min } \rightarrow \infty\right)$, and 4) constant acceleration of the preceding vehicle, defined by $a_{p}(t)=a_{p}(0)=a_{p .0}$ for $t \in\left[0, t_{p}\right]$, where $a_{p .0}$ is updated at every time step. 


\section{A. Unconstrained Case}

In absence of state inequality constraints, Hamiltonian function is first formed as

$$
H=b_{1} v u+b_{2} u^{2}+\lambda_{1} v+\lambda_{2}\left(c_{1} u-c_{0}\right),
$$

where $\lambda_{1}$ and $\lambda_{2}$ are position and speed co-state variables, respectively.

Then, the necessary optimality conditions (Pontryagin's minimum principle) are used to derive a two-point boundary value problem. Following the derivation in [23], the optimal control input can be expressed as a linear function of time,

$$
u^{*}(t)=k_{1} t+k_{2},
$$

where $k_{1}=\left(b_{1} c_{0}+c_{1} \lambda_{1.0}\right) /\left(2 b_{2}\right), \quad k_{2}=-\left(b_{1} v_{0}+\right.$ $\left.c_{1} \lambda_{2.0}\right) /\left(2 b_{2}\right)$, with $\lambda_{1}^{*}(0)=\lambda_{1.0}$ and $\lambda_{2}^{*}(0)=\lambda_{2.0}$.

Using (16), the system dynamics can be integrated. Then, enforcing the terminal constraints, $s^{*}\left(t_{p}\right)=S, v^{*}\left(t_{p}\right)=V$, a system of two linear equations in two unknowns $\left(\lambda_{1.0}\right.$ and $\left.\lambda_{2.0}\right)$ is obtained as

$$
\left[\begin{array}{l}
S \\
V
\end{array}\right]=\left[\begin{array}{l}
s^{*}\left(t_{p}\right) \\
v^{*}\left(t_{p}\right)
\end{array}\right]=A\left[\begin{array}{l}
\lambda_{1.0} \\
\lambda_{2.0}
\end{array}\right]+B
$$

where

$$
A=\frac{c_{1}^{2} t_{p}}{12 b_{2}}\left[\begin{array}{cc}
t_{p}^{2} & -3 t_{p} \\
3 t_{p} & -6
\end{array}\right], B=\frac{1}{12 b_{2}}\left[\begin{array}{c}
B_{1} \\
B_{2}
\end{array}\right],
$$

while $B_{1}=b_{1} c_{0} c_{1} t_{p}^{3}-3\left(2 b_{2} c_{0}+b_{1} c_{1} v_{0}\right) t_{p}^{2}+12 b_{2}\left(v_{0} t_{p}+s_{0}\right)$ and $B_{2}=3 b_{1} c_{0} c_{1} t_{p}^{2}-6\left(2 b_{2} c_{0}+b_{1} c_{1} v_{0}\right) t_{p}+12 b_{2} v_{0}$.

By solving it, the optimal speed trajectory is obtained as a quadratic function of time (parabola), where the details are given in [14].

\section{B. State-constrained Case}

Constraints in (12a) and (12b) are pure state inequality constraints of the form $h(x, t) \leq 0$, where $x$ denotes the state variables, therefore are not directly dependent on the control variable. If $h(x, t)=0$ for $t \in\left[t_{1}, t_{2}\right]$ with $t_{1}<t_{2}$, this interval is called boundary interval, where the time, $t_{1}$ (or $t_{2}$ ), to start (or end) the boundary interval is called an entry time (or exit time). Furthermore, if the state trajectory touches the boundary, this specific time is called a contact time. The entry, exit, and contact times are called junction times.

Generally, there are two methods to handle the pure state inequality constraints: the direct and the indirect adjoining methods [24]. Of the two, the indirect adjoining method is used in this work. If $h(x, t)$ is of pth order, it is differentiated $p$ times with respect to time until the control variable explicitly appears, and then $h^{(p)}(x, u, t)$ is adjoined to the Hamiltonian with a multiplier $\mu$ to form the Lagrangian,

$$
L(x, u, t)=H(x, u, t)+\mu h^{(p)}(x, u, t),
$$

where $\mu h(x, t)=0, \mu \geq 0$.

Imposing only $h^{(p)}(x, u, t) \leq 0$ whenever $h(x, t)=0$ does not prevent the trajectory from violating $h(x, t) \leq 0$ because it cannot guarantee that $h^{(q)}(x, t) \leq 0$ for $q=1, \cdots, p-1$. From this fact, tangency conditions, $\Psi=\left[h^{(0)}, h^{(1)}, \cdots, h^{(p-1)}\right]^{T}=$ 0 , must be added at the entry time [25]. Because the tangency conditions form interior-point constraints, the necessary optimality conditions are

$$
\begin{aligned}
& u^{*}(t)=\arg \min _{u \in \Omega\left(x^{*}, t\right)} H\left(u^{*}, x^{*}, \lambda^{*}, t\right), \\
& \dot{x}^{*}(t)=L_{\lambda}^{*}\left(u^{*}, x^{*}, \lambda^{*}, \mu^{*}, t\right), \\
& \dot{\lambda}^{*}(t)=L_{x}^{*}\left(u^{*}, x^{*}, \lambda^{*}, \mu^{*}, t\right),
\end{aligned}
$$

where $\mu^{*} h^{(p)}=0, \mu^{*} \geq 0$, and $\Omega=\left\{h^{(p)} \leq 0\right.$ if $\left.h=0\right\}$, while $\lambda$ denotes co-state variables.

To satisfy the tangency conditions, the co-state variable may be discontinuous at the entry time according to the following jump conditions,

$$
\begin{aligned}
& \lambda^{*}\left(\tau^{-}\right)=\lambda^{*}\left(\tau^{+}\right)+\sum_{j=0}^{p-1} \pi_{j} h_{x^{*}}^{(j)}\left(x^{*}, \tau\right), \\
& H\left(\tau^{-}\right)=H\left(\tau^{+}\right)-\sum_{j=0}^{p-1} \pi_{j} h_{t}^{(j)}\left(x^{*}, \tau\right),
\end{aligned}
$$

where $\tau \in\left[0, t_{p}\right]$ indicates the entry time, and $\pi_{j}(j=$ $0, \cdots, p-1)$ are multipliers for the tangency conditions.

To apply the above analytical approach to the safe- and eco-driving control problem, the preceding vehicle's position must be predicted for all $t \in\left[0, t_{p}\right]$. The position constraint is rewritten using the assumption of constant acceleration,

$$
h_{2}(t)=s(t)-\left(s_{p .0}+v_{p .0} t+a_{p .0} t^{2} / 2\right),
$$

where $v_{p}(t)=v_{p .0}+a_{p .0} t$, while $s_{p}(0)=s_{p .0}$ and $v_{p}(0)=$ $v_{p .0}$ are the preceding vehicle's position and speed measured at $t=0$. Note that $\delta_{s}$ is lumped in $s_{p .0}$.

The speed and position constraints are of the first order $\left(p_{1}=1\right)$ and of the second order $\left(p_{2}=2\right)$, respectively, where $h_{1}^{(1)}=c_{1} u-c_{0}$ and $h_{2}^{(2)}=c_{1} u-c_{0}-a_{p .0}$. The resulting tangency conditions are

$$
\begin{aligned}
\Psi_{1} & =v(\tau)-v_{\max }=0, \\
\Psi_{2} & =\left[\begin{array}{c}
s(\tau)-\left(s_{p .0}+v_{p .0} \tau+a_{p .0} \tau^{2} / 2\right) \\
v(\tau)-\left(a_{p .0} \tau+v_{p .0}\right)
\end{array}\right]=0,
\end{aligned}
$$

where $\tau \in\left[0, t_{p}\right]$ represents entry time among junction times.

The Lagrangian is formed as

$$
L=H+\mu_{1}\left(c_{1} u-c_{0}\right)+\mu_{2}\left(c_{1} u-c_{0}-a_{p .0}\right),
$$

where $\mu_{i}=0$ if $h_{i}<0, \mu_{i} \geq 0$ if $h_{i}=0$ for $i=1,2$. Note that the values of $\mu_{i}$ are always zero because of the continuous optimal control input.

The jump conditions of the co-state variables are summarized as follows. In the case of the first-order speed constraint,

$$
\begin{aligned}
\lambda_{1}^{*}\left(\tau^{-}\right) & =\lambda_{1}^{*}\left(\tau^{+}\right), \\
\lambda_{2}^{*}\left(\tau^{-}\right) & =\lambda_{2}^{*}\left(\tau^{+}\right)+\pi_{0 . h_{1}}, \\
H\left(\tau^{-}\right) & =H\left(\tau^{+}\right),
\end{aligned}
$$

where $\pi_{0 . h_{1}}$ is a multiplier of $h_{1}^{(0)}$. In the case of the secondorder position constraint,

$$
\begin{aligned}
\lambda_{1}^{*}\left(\tau^{-}\right) & =\lambda_{1}^{*}\left(\tau^{+}\right)+\pi_{0 . h_{2}}, \\
\lambda_{2}^{*}\left(\tau^{-}\right) & =\lambda_{2}^{*}\left(\tau^{+}\right)+\pi_{1 . h_{2}}, \\
H\left(\tau^{-}\right) & =H\left(\tau^{+}\right)+\pi_{0 . h_{2}}\left(a_{p .0} \tau+v_{p .0}\right)+\pi_{1 . h_{2}} a_{p .0},
\end{aligned}
$$


where $\pi_{0 . h_{2}}$ and $\pi_{1 . h_{2}}$ are the multipliers of $h_{2}^{(0)}$ and $h_{2}^{(1)}$, respectively.

In both cases, the jump parameters of speed co-state $\left(\pi_{0 . h_{1}}\right.$ and $\left.\pi_{1 . h_{2}}\right)$ are always zero because of the continuous optimal control input. Furthermore, the active speed constraint does not have a jump parameter of position co-state, and thus both co-state variables are continuous without any jumps. On the other hand, the position co-state in case of an active position constraint must be discontinuous at the entry time because of the non-zero jump parameter $\left(\pi_{0 . h_{2}} \neq 0\right)$.

There are three cases to consider depending on which state constraint is active.

1) Speed-Only-Constrained Case. If the speed of the unconstrained solution exceeds the maximum road speed limit, a speed-constrained optimal solution must be computed. This optimal control is defined by three phases,

$$
u^{*}(t)=\left\{\begin{array}{cc}
k_{1} t+k_{2} & {\left[0, t_{1}\right)} \\
u_{c . v} & {\left[t_{1}, t_{2}\right),} \\
k_{1}\left(t-t_{2}\right)+u_{c . v} & {\left[t_{2}, t_{p}\right]}
\end{array}\right.
$$

where $k_{1}=\left(b_{1} c_{0}+c_{1} \lambda_{1.0}\right) /\left(2 b_{2}\right), \quad k_{2}=-\left(b_{1} v_{0}+\right.$ $\left.c_{1} \lambda_{2.0}\right) /\left(2 b_{2}\right)$. The boundary control input, $u_{c . v}=c_{0} / c_{1}$, is defined by the condition on the boundary interval, $h_{1}^{(1)}=0$.

A system of four nonlinear equations $\left(u^{*}\left(t_{1}\right)=u_{c . v}\right.$, $v^{*}\left(t_{1}\right)=v_{\max }, v^{*}\left(t_{p}\right)=V$, and $s^{*}\left(t_{p}\right)=S$ ) in four unknowns $\left(\lambda_{1.0}, \lambda_{2.0}, t_{1}\right.$, and $\left.t_{2}\right)$ is obtained; a solution to this system is

$$
\begin{gathered}
A_{1.1} t_{1}^{2}+A_{1.2} t_{1}+A_{1.3}=0, \\
t_{2}=\left(B_{1.1} t_{1}+B_{1.2}\right) / B_{1.3},
\end{gathered}
$$

for $0<t_{1}<t_{2}<t_{p}$, with

$$
\begin{aligned}
& \lambda_{1.0}=C_{1.1}+C_{1.2} / t_{1}^{2}, \\
& \lambda_{2.0}=D_{1.1}+D_{1.2} / t_{1},
\end{aligned}
$$

where coefficients $A$ 's, $B$ 's, $C$ 's, and $D$ 's are given in Appendix A.

2) Position-Only-Constrained Case. If the inter-vehicle distance with the unconstrained solution is smaller than the minimum safe distance, a position-only-constrained solution must be computed. As mentioned above, the position constraint becomes active either on the boundary interval or at the contact point depending on the activation condition of the mixed state inequality constraints, $h_{2}^{(2)} \leq 0$. In the case of the boundary interval, the optimal control is defined by three phases,

$$
u^{*}(t)=\left\{\begin{array}{cc}
k_{1} t+k_{2} & {\left[0, t_{1}\right)} \\
u_{c . s} & {\left[t_{1}, t_{2}\right)} \\
k_{3}\left(t-t_{2}\right)+u_{c . s} & {\left[t_{2}, t_{p}\right]}
\end{array}\right.
$$

where $k_{1}$ and $k_{2}$ have the same definition as in the previous section, and $k_{3}=k_{1}+c_{1} \pi_{0 . h_{2}} /\left(2 b_{2}\right)$. The boundary control input $u_{c . s}=\left(c_{0}+a_{p .0}\right) / c_{1}$ is defined by the condition on the boundary interval, $h_{2}^{(2)}=0$.

A system of five nonlinear equations $\left(u^{*}\left(t_{1}\right)=u_{c . s}\right.$, $v^{*}\left(t_{1}\right)=v_{p}\left(t_{1}\right), s^{*}\left(t_{1}\right)=s_{p}\left(t_{1}\right), v^{*}\left(t_{p}\right)=V$, and $s^{*}\left(t_{p}\right)=$ $S)$ in five unknowns $\left(\lambda_{1.0}, \lambda_{2.0}, t_{1}, t_{2}\right.$, and $\left.\pi_{0 . h_{2}}\right)$ is obtained; a solution to this system is

$$
\begin{aligned}
& t_{1}=A_{2 . b .1} / A_{2 . b .2}, \\
& t_{2}=B_{2 . b .1} / B_{2 . b .2},
\end{aligned}
$$

for $0<t_{1}<t_{2}<t_{p}$, with

$$
\begin{aligned}
\lambda_{1.0} & =C_{2 . b .1}+C_{2 . b .2} / t_{1}^{2}, \\
\lambda_{2.0} & =D_{2 . b .1}+D_{2 . b .2} / t_{1}, \\
\pi_{0 . h_{2}} & =\frac{E_{2 . b .1}+\left(E_{2 . b .2} t_{2}^{2}+E_{2 . b .3} t_{2}+E_{2 . b .4}\right) / t_{1}^{2}}{\left(t_{2}-t_{p}\right)^{2}}
\end{aligned}
$$

where coefficients $A$ 's, $B$ 's, $C$ 's, $D$ 's, and $E$ 's are given in Appendix B-1.

As the activation level of the position constraint becomes looser, the boundary interval vanishes and becomes a contact point. In this case, $h_{2}^{(2)}<0$ is always satisfied, thus the resulting optimal control has only two phases,

$$
u^{*}(t)=\left\{\begin{array}{cc}
k_{1} t+k_{2} & {\left[0, t_{1}\right)} \\
k_{3}\left(t-t_{1}\right)+u^{*}\left(t_{1}\right) & {\left[t_{1}, t_{p}\right]}
\end{array},\right.
$$

where $k_{1}, k_{2}$, and $k_{3}$ have the same definition as in the previous section.

A system of four nonlinear equations $\left(v^{*}\left(t_{1}\right)=v_{p}\left(t_{1}\right)\right.$, $s^{*}\left(t_{1}\right)=s_{p}\left(t_{1}\right), v^{*}\left(t_{p}\right)=V$, and $s^{*}\left(t_{p}\right)=S$ ) in four unknowns $\left(\lambda_{1.0}, \lambda_{2.0}, t_{1}\right.$, and $\left.\pi_{0 . h_{2}}\right)$ is obtained; a solution to this system is

$$
A_{2 . c .1} t_{1}^{3}+A_{2 . c .2} t_{1}^{2}+A_{2 . c .3} t_{1}+A_{2 . c .4}=0,
$$

for $0<t_{1}<t_{p}$, with

$$
\begin{aligned}
\lambda_{1.0} & =C_{2 . c .1}+C_{2 . c .2} / t_{1}^{2}+C_{2 . c .3} / t_{1}^{3}, \\
\lambda_{2.0} & =D_{2 . c .1}+D_{2 . c .2} / t_{1}+D_{2 . c .3} / t_{1}^{2}, \\
\pi_{0 . h_{2}} & =\frac{E_{2 . c .1}+E_{2 . c .2} / t_{1}+E_{2 . c .3} / t_{1}^{2}+E_{2 . c .4} / t_{1}^{3}}{\left(t_{1}-t_{p}\right)^{2}},
\end{aligned}
$$

where coefficients $A$ 's, $B$ 's, $C$ 's, $D$ 's, and $E$ 's are given in Appendix B-2.

3) Both Speed-and Position-Constrained Case. There are several cases depending on the active sequence of the two constraints. For example, if the speed constraint is firstly active on the boundary interval $\left(t \in\left[t_{1.1}, t_{1.2}\right]\right)$ and then the position constraint is active on the boundary interval $\left(t \in\left[t_{2.1}, t_{2.2}\right]\right)$, the corresponding optimal control is formed as,

$$
u^{*}(t)=\left\{\begin{array}{cc}
k_{1} t+k_{2} & {\left[0, t_{1.1}\right)} \\
u_{c . v} & {\left[t_{1.1}, t_{1.2}\right)} \\
k_{1}\left(t-t_{1.2}\right)+u_{c . v} & {\left[t_{1.2}, t_{2.1}\right)} \\
u_{c . s} & {\left[t_{2.1}, t_{2.2}\right)} \\
k_{3}\left(t-t_{2.2}\right)+u_{c . s} & {\left[t_{2.2}, t_{p}\right]}
\end{array}\right.
$$

and a system of seven nonlinear equations $\left(u^{*}\left(t_{1.1}\right)=u_{c . v}\right.$, $v^{*}\left(t_{1.1}\right)=v_{\max }, u^{*}\left(t_{2.1}\right)=u_{c . s}, v^{*}\left(t_{2.1}\right)=v_{p}\left(t_{2.1}\right)$, $s^{*}\left(t_{2.1}\right)=s_{p}\left(t_{2.1}\right), v^{*}\left(t_{p}\right)=V$, and $\left.s^{*}\left(t_{p}\right)=S\right)$ in seven unknowns $\left(\lambda_{1.0}, \lambda_{2.0}, t_{1.1}, t_{1.2}, t_{2.1}, \pi_{0 . h_{2}}\right.$, and $\left.t_{2.2}\right)$ is obtained and solved.

Analogously, the speed- and position-constrained solution of other cases can be computed.

\section{Conditions for Feasible Analytical Solution}

Terminal conditions at final time determine whether the analytical state-constrained solution exists or not. A feasible terminal speed condition is easily defined by the requirement that a terminal speed must be lower than the maximum speed 
limit. On the other hand, a feasible terminal position is affected by the preceding vehicle's driving as well as the maximum speed limit, thus such a condition is more critical to set. Consider two consecutive vehicles driving on the same lane; the host vehicle does not overtake the preceding vehicle or change the direction of movement on the planned route. From this point of view, the terminal position condition, a pair $\left(t_{p}, S\right)$, may be infeasible under two types of scenarios: a stop (Fig. 1(a)) and non-stop scenario (Fig. 1(b)). In the first scenario, the preceding vehicle will stop before arriving at $S$, whereas in the second scenario, it will drive too slow to arrive at $S$ within $t_{p}$. Therefore, it is necessary to define a feasible range of the terminal position and to guarantee the existence of the state-constrained solution.

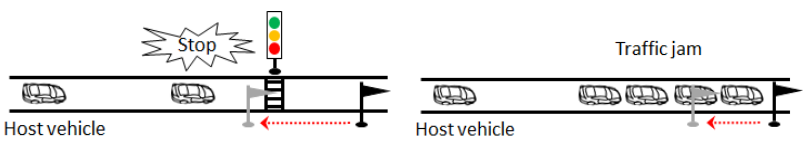

Fig. 1. Two scenarios for non-existence of analytical solution: stop scenario (a) and non-stop scenario (b).

\section{A. Maximum Terminal Position}

An active state constraint imposes the maximum terminal position that the host vehicle can reach at the end of the prediction horizon. There are three definitions of the maximum terminal position depending on which state constraint becomes active: 1) the speed constraint, 2) the position constraint, and 3) both of them.

In the first case, an active speed constraint generates its boundary interval and then penalizes the terminal position of the host vehicle. If this boundary interval expands and equals the prediction horizon, the resulting travel distance sets a condition for $S$,

$$
S \leq S_{\operatorname{max.1}}\left(t_{p}\right)=s_{0}+v_{\max } t_{p}
$$

In the second case of an active position constraint, the resulting travel distance of the preceding vehicle sets a condition for $S$,

$$
S \leq S_{\max .2}\left(t_{p}\right)=s_{p .0}+v_{p .0} t_{p}+a_{p .0} t_{p}^{2} / 2 .
$$

In the last case, the two constraints are active in sequence. If the speed constraint precedes the position constraint, (44) is still valid. However, if the position constraint precedes the speed constraint, the terminal position is more penalized than that in (44). In the extreme situation when the exit (or contact) time of the position constraint equals the entry time of the speed constraint, and the exit time of the speed constraint equals the prediction horizon, the resulting travel distance indicates the maximum terminal position. In this case, if the entry time of the speed constraint is smaller than the prediction horizon $\left(t_{1.1} \leq t_{p}\right)$, the additional condition for $S$ is

$$
\begin{aligned}
S \leq S_{\max .3}\left(t_{p}\right) & =s_{p}\left(t_{1.1}\right)+v_{\max }\left(t_{p}-t_{1.1}\right) \\
& =s_{p .0}-\frac{\left(v_{\max }-v_{p .0}\right)^{2}}{2 a_{p .0}}+v_{\max } t_{p}
\end{aligned}
$$

where $t_{1.1}:=\left(v_{\max }-v_{p .0}\right) / a_{p .0}$, obtained from $v_{p}\left(t_{1.1}\right)=$ $v_{\max }$.

In summary, the maximum terminal position is written as

$$
S_{\max }\left(t_{p}\right)=\min \left(S_{\max .1}, S_{\max .2}, S_{\max .3}\right),
$$

where the most limiting conditions (min) depends on $t_{p}$.

A first threshold can be computed imposing $S_{\text {max.1 }}\left(t_{\text {p.th.1 }}\right)=S_{\operatorname{max.2}}\left(t_{\text {p.th.1 }}\right)$ and solving the following equation,

$$
F_{1}+F_{2} t_{p . t h .1}+F_{3} t_{p . t h .1}^{2}=0,
$$

where $F_{1}=s_{0}-s_{p .0}, F_{2}=v_{\max }-v_{p .0}, F_{3}=-a_{p .0} / 2$.

The second threshold is the entry time of the speed constraint,

$$
t_{p . t h .2}=t_{1.1}
$$

If there exist $t_{p . t h .1}$ and $t_{p . t h .2}$ satisfying

$$
0<t_{p . t h .1}<t_{p . t h .2}
$$

the maximum terminal position can be written as

$$
S_{\max }\left(t_{p}\right)=\left\{\begin{array}{cc}
S_{\max .1} & t_{p} \in\left[0, t_{p . t h .1}\right) \\
S_{\max .2} & t_{p} \in\left[t_{p . t h .1}, t_{p . t h .2}\right) \\
S_{\max .3} & t_{p} \in\left[t_{p . t h .2}, \infty\right]
\end{array}\right\} .
$$

\section{B. Minimum Terminal Position}

In case of a small travel distance, the unconstrained solution might cause the host vehicle to drive backward in the vicinity of the end of the prediction horizon. This is mainly due to the fact that the optimal speed profile is a quadratic function of time, and this function, with a zero terminal speed, changes from being concave to convex as the terminal position decreases. The limit feasible case is when the speed profile becomes linear, i.e., $k_{1}=0$ in (16), the corresponding speed and position are $v^{l}(t)=v_{0}-v_{0} t / t_{p}$ and $s^{l}(t)=s_{0}+v_{0} t-v_{0} t^{2} /\left(2 t_{p}\right)$. There are two definitions of the minimum terminal position depending on whether the position constraint becomes inactive or active. In the first case, the minimum terminal position is defined as

$$
S_{\text {min. } 1}\left(t_{p}\right)=s^{l}\left(t_{p}\right)=s_{0}+v_{0} t_{p} / 2 .
$$

In the second case, the contact time, $t_{c}$, such that $v^{*}\left(t_{c}\right)=$ $v_{p}\left(t_{c}\right)$ and $s^{*}\left(t_{c}\right)=s_{p}\left(t_{c}\right)$ exists in order to enforce the position constraint. This position-constrained solution having
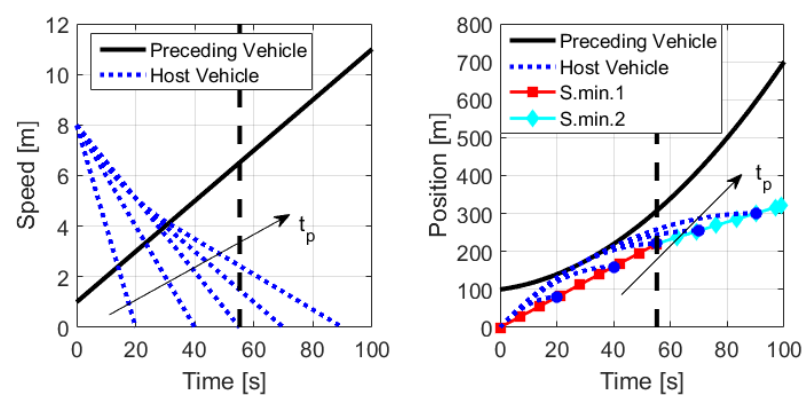

Fig. 2. Trajectories of the host vehicle generating the minimum terminal position for five different values of $t_{p}$ : speed (a) and position (b). Bold black line is the trajectory of the preceding vehicle, and dashed black line is $t_{p . t h .3}$. 
linear speed profile after $t_{c}$ results in the minimum terminal position, as follows:

$$
\begin{aligned}
S_{\text {min.2 }}\left(t_{p}\right) & =s_{p}\left(t_{c}\right)+v_{p}\left(t_{c}\right)\left(t_{p}-t_{c}\right), \\
& =s_{p .0}+\frac{v_{p .0} t_{c}}{2}+\frac{v_{p .0}+a_{p .0} t_{c}}{2} t_{p},
\end{aligned}
$$

and $t_{c}$ is computed imposing $k_{3}=\left(b_{1} c_{0}+c_{1}\left(\lambda_{1.0}+\right.\right.$ $\left.\left.\pi_{1 . p}\right)\right) /\left(2 b_{2}\right)=0$ in (34) and solving the following equation,

$$
F_{5} t_{c}^{2}+F_{6} t_{c}+F_{7}=0 \text { for } 0<t_{c}<t_{p . s},
$$

where $F_{5}=2 v_{0}-3 v_{p .0}-a_{p .0} t_{p . s}, F_{6}=6\left(s_{0}-s_{p .0}\right)+$ $2 t_{p . s}\left(v_{p .0}-v_{0}\right), F_{7}=6 t_{p . s}\left(s_{p .0}-s_{0}\right)$.

The threshold that activates the position constraint, $t_{\text {p.th.3 }}$, exists only if there exists the touch point such that $s^{l}\left(t_{u n . c}\right)=$ $s_{p}\left(t_{\text {un.c }}\right)=0$. Using the discriminant of the condition for $t_{\text {un.c }}, t_{p . t h .3}$ is written as

$$
t_{p . t h .3}=\frac{2\left(s_{p .0}-s_{0}\right) v_{0}}{\left(v_{p .0}-v_{0}\right)^{2}-2 a_{p .0}\left(s_{p .0}-s_{0}\right)} .
$$

With $t_{p . t h .3}$, the minimum terminal position can be written as

$$
S_{\text {min }}\left(t_{p}\right)=\left\{\begin{array}{ll}
S_{\text {min.1 }} & t_{p} \in\left[0, t_{p . t h .3}\right) \\
S_{\text {min.2 }} & t_{p} \in\left[t_{p . t h .3}, \infty\right]
\end{array}\right\} .
$$

Figure 2 shows that the speed trajectory becomes a linear function over the whole horizon or the sub-horizon if the position constraint is active. Moreover, the corresponding terminal position points build up the minimum terminal position curve $\left(S_{\min }\left(t_{p}\right)\right)$.

\section{Feasible Range}

In this section, the conditions on terminal position are illustrated using examples of normal and abnormal scenarios. The feasible range is defined as the area in the plane $\left(t_{p}, S\right)$ between the maximum and minimum terminal position curves as shown in Fig. 3. Suppose that the point $\left(t_{p}, S\right)$ was set to $\left(t_{p . s}, S_{s}\right)$ ("Set point"). In the normal scenario, the set point is a feasible terminal condition, whereas in the abnormal scenarios, it becomes an infeasible terminal condition and thus must be corrected to be in the feasible range as $\left(t_{p . a}, S_{a}\right)$ ("Adjusted point"). In the non-stop scenario, only the terminal position must be adjusted, whereas in the stop scenario, the prediction horizon must be also shrunk. In other terms, when the preceding vehicle is braking suddenly and sharply (stop scenario), the host vehicle must take an action to stop itself optimally considering the stopping distance and time of the preceding vehicle.

The adjusted terminal position must be as far as possible to avoid large torque values afterwards that cause unnecessary energy losses. This adaptation of the terminal position condition guarantees the existence of the analytical solution, and thus improves robustness of the MPC with respect to uncertain driving of the preceding vehicle.

\section{Simulation}

\section{A. Simulation Environment}

The preceding vehicle is assumed to drive a real-world speed profile, and its future driving behavior is only predicted using the current state information. To evaluate the proposed safe- and eco-driving control system, three speed profiles are extracted from experimental data of actual trips in the city of Aachen, Germany, [26] (urban driving scenario), while the Artemis highway driving cycle is selected as a highway driving scenario, see Fig. 4. The initial position of the preceding vehicle $\left(s_{p .0}\right)$ is set to $50 \mathrm{~m}$. Vehicle parameters are listed in Table I. Simulation is performed on a standard desktop computer with a $3.50 \mathrm{GHz}$ Intel quad core chip and 16.0 GB RAM using MATLAB 2015b.

TABLE I

VEHICLE SPECIFICATIONS

\begin{tabular}{c||c|cc}
\hline & Category & Value & Unit \\
\hline \hline \multirow{4}{*}{ Vehicle } & Total mass, $m$ & 1432 & $\mathrm{~kg}$ \\
& Wheel radius, $r$ & 0.2820 & $\mathrm{~m}$ \\
& Frontal area, $A_{f}$ & 1.1536 & $\mathrm{~m}^{2}$ \\
& Aerodynamic drag coefficient, $c_{d}$ & 0.44 & - \\
& Ambient air density, $\rho_{a}$ & 1.18 & $\mathrm{kgm}^{-3}$ \\
& Rolling resistance coefficient, $c_{r}$ & 0.0132 & - \\
\hline Transmission & Transmission ratio, $R_{t}$ & 9.59 & - \\
& Transmission efficiency, $\eta_{t}$ & 0.98 & - \\
\hline Electric motor & $b_{1}=R_{t} / r$ & 34.007 & $\mathrm{~m}^{-1}$ \\
& Loss coefficient, $b_{2}$ & 0.8730 & \\
\hline
\end{tabular}

\section{B. Controller Setup}

A desired arrival time and position are set to $T_{0}$ and $S_{0}$, respectively. At every time step, the MPC updates current time and position $\left(t_{0}\right.$ and $\left.s_{0}\right)$. If the prediction horizon is initially
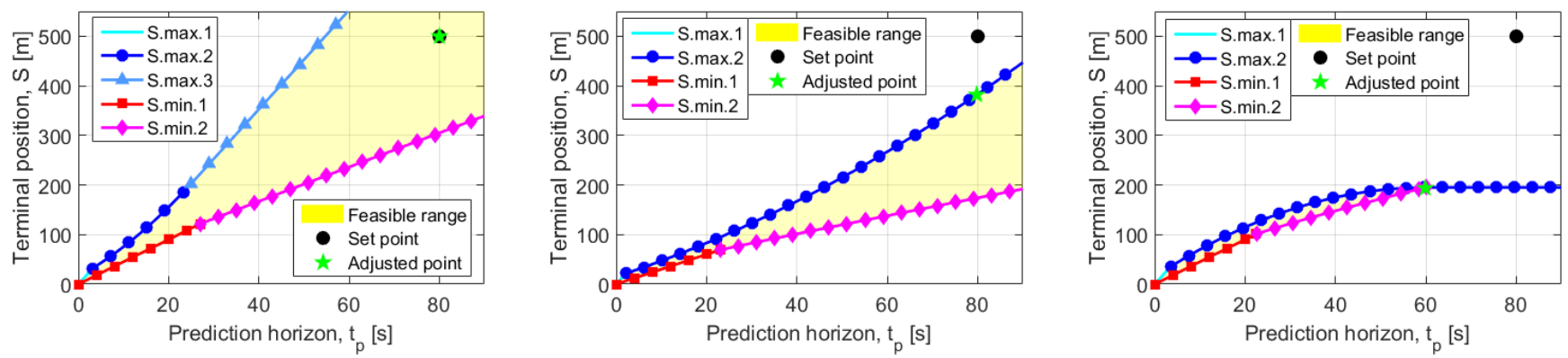

Fig. 3. Feasible range of the terminal position with $\left(t_{p . s}, S_{s}\right)=(80,500)$ for three scenarios: normal (a), abnormal non-stop (b), and abnormal stop (c). 

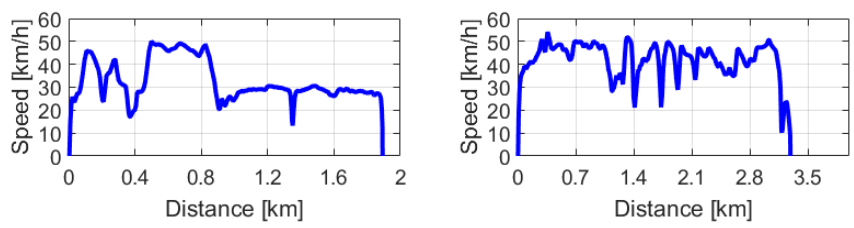

Fig. 4. Four driving scenarios for the preceding vehicle

set to $T_{p}<T_{0}$, the $T_{p}$ is not changed unless a remaining travel time $\left(T_{0}-t_{0}\right)$ becomes smaller than it. However, if $T_{p}>T_{0}$, the desired arrival time is not $T_{0}$, but $T_{p}$. Therefore, the set value of the prediction horizon, $t_{p . s}$ is

$$
t_{p . s}\left(t_{0}\right)=\left\{\begin{array}{cc}
\min \left(T_{p}, T_{0}-t_{0}\right) & T_{p}<T_{0} \\
T_{p}-t_{0} & T_{p} \geq T_{0}
\end{array}\right\}
$$

The shorter the used $t_{p . s}$ is, the more prone to be an infeasible terminal position $S_{0}$ is. Although the terminal position is adjusted in the feasible range, large control input values are inevitably generated to cover this distance. For this reason, it is necessary to change the desired terminal position depending on $t_{p . s}$. Therefore, the desired mean speed, $v_{\text {mean }}\left(t_{0}\right)=\left(S_{0}-s_{0}\right) /\left(T_{0}-t_{0}\right)$, is used to define a set value of terminal position as

$$
\begin{aligned}
S_{s}\left(t_{0}\right) & =\min \left(S_{0}, s_{0}+v_{\text {mean }} t_{p . s}\right), \\
& =\min \left(S_{0}, s_{0}+\left(S_{0}-s_{0}\right) /\left(T_{0}-t_{0}\right) t_{p . s}\right) .
\end{aligned}
$$

Depending on whether the terminal conditions $\left(t_{p . s}, S_{s}\right)$ are feasible or not, the adjusted terminal conditions are

$$
\left(t_{p}, S\right)=\left\{\begin{array}{cc}
\left(t_{p . s}, S_{s}\right) & \text { if feasible } \\
\left(t_{p . s}, S_{a}\right) \text { or }\left(t_{p . a}, S_{a}\right) & \text { else }
\end{array}\right\}
$$

The proposed MPC computes the closed-form optimal control input to cover the distance $S$ in a $t_{p}$ time and applies the first computed control input to drive the electric CAV. The MPC updating period, at which the MPC computes a new optimal control input, is set to $0.1 \mathrm{~s}$. The maximum speed limit is set to the maximum speed of the preceding vehicle (i.e. $v_{\max }=[51,58,90,150] \mathrm{km} / \mathrm{h}$ ) and the minimum safe distance $\left(\delta_{s}\right)$ is set to $5 \mathrm{~m}$.

\section{Performance Evaluation}

In this section, the proposed MPC is evaluated in terms of real-time computation capability and energy consumption optimality. As a measure of computation time, the mean time to generate the analytical solution at every time step is used. The energy consumption over the whole trip, $E_{f}$, is calculated with the full model in (1)-(4). The reference optimal energy consumption, $E_{\text {f.opt }}$, is computed using an interior penalty method [27] and a collocation method (bvp5c in MATLAB [28]) to solve the state-constrained optimal control problem with a perfect knowledge about future driving of the preceding vehicle. The loss of energy optimality is defined as

$$
\mathrm{LoO}=\left(E_{f}-E_{f . o p t}\right) / E_{f . o p t} \times 100 .
$$

The LoO of the preceding vehicle is also computed in the same way for comparison.
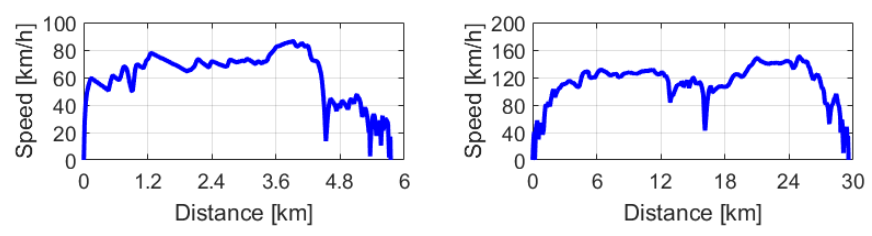

1) Effect of Prediction Horizon $\left(T_{p}\right)$ : Figure 5(a) shows the variation of the final energy consumption with $T_{p}$ for the second scenario of Fig. 4. Generally, as $T_{p}$ increases to $T_{0}$, the energy consumption decreases, however the curve $E_{f}\left(T_{p}\right)$ has a minimum point on the left of $T_{0}$, where optimized $T_{p}$ generating the minimum is denoted by $T_{p}^{*}$. In the right region, $T_{p}$ is also the desired arrival time (see 56), thus as $T_{p}$ increases, the resulting final energy consumption monotonically decreases. As for the real-time computation capability, the computing time generally increases with $T_{p}$, but it is generally small enough to implement on an on-board controller (less than $20 \mathrm{~ms}$ in general and $4 \mathrm{~ms}$ when $T_{p}^{*}$ is used), as shown in Fig. 5(b).
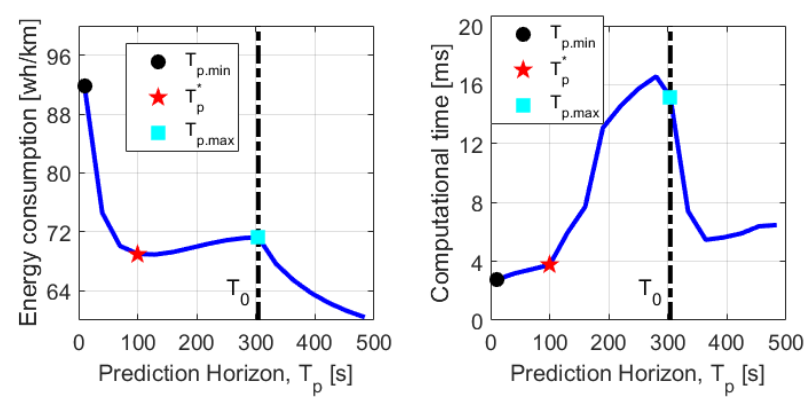

Fig. 5. Effect of the initial prediction horizon on energy consumption (a) and computational time (b), for the second driving scenario.

Figure 6 shows the trajectories for three different values of $T_{p}$ : short $\left(T_{p . \text { min }}=10 \mathrm{~s}\right)$, optimized $\left(T_{p}^{*}=100 \mathrm{~s}\right)$, long $\left(T_{p \cdot \max }=T_{0}=304 \mathrm{~s}\right)$. The shorter prediction horizon generates almost the same speed as the preceding vehicle, while avoiding a rear-end collision (Fig. 6(a)). For this reason, sharp and large control inputs are generated and thus the resulting energy consumption is only slightly reduced with respect to the preceding vehicle. On the other hand, in the other cases, the preceding vehicle driving behavior can be predicted over a sufficiently long horizon, and thus the increase/decrease in the speed is closer to optimum. As shown in Fig. 6(c), the optimal control input is smoother in these cases than in the case of a shorter prediction horizon. This is the main reason of the significant improvement in terms of energy consumption (Fig. 5(a) and Fig. 6(d)).

On the other hand, the longer prediction horizon tends to increase the speed slowly in the beginning of the trip due to aerodynamic drag resistance which is not considered in the analytical solution. Then, it must reach and cruise the maximum speed in order to arrive at destination on time, which results in some loss of energy optimality. This is the same reason for the convex curve of $E_{f}\left(T_{p}\right)$ in Fig. 5(a). 

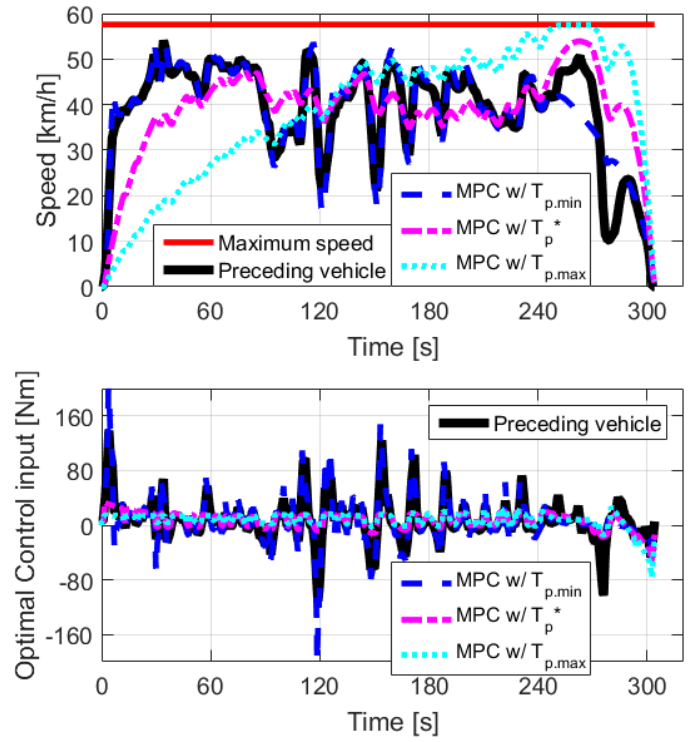
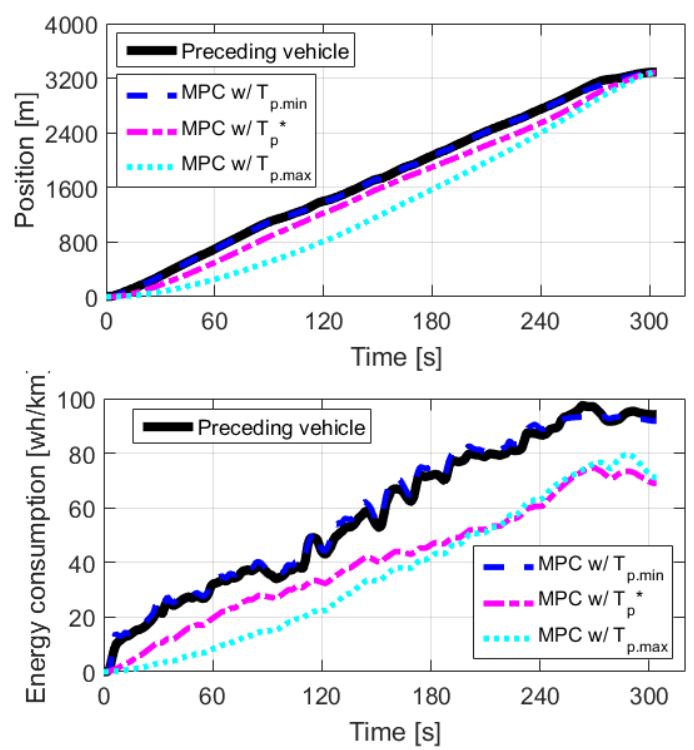

Fig. 6. Trajectories for three different values of initial prediction horizon for the second driving scenario: speed (a), position (b), optimal control input (c), and energy consumption (d).

These results lead to the observation that a short prediction horizon can maintain small inter-vehicle distance by mimicking the speed of the preceding vehicle; it means that the proposed system can also serve as energy-efficient ACC by keeping a small prediction horizon.

2) Effect of Minimum Safe Distance $\left(\delta_{s}\right)$ : The difference $\left(s_{p .0}-\delta_{s}\right)$ in the position constraint (6) fixes the position boundary. Thus, this section analyzes the effect of another control parameter, $\delta_{s}$, on the energy consumption as well as the inter-vehicle gap. Besides $\delta_{s}=5 \mathrm{~m}$, three different values, $\delta_{s}=[10,30,50] \mathrm{m}$, are used. As shown in Fig. 7, as $\delta_{s}$ increases up to $s_{p .0}$ the position constraint becomes more aggressive and consequently the energy consumption increases. Note, however, that the increase in energy consumption caused by the increase in $\delta_{s}$ is very small. As for safe driving, intervehicle gap increases with $\delta_{s}$.
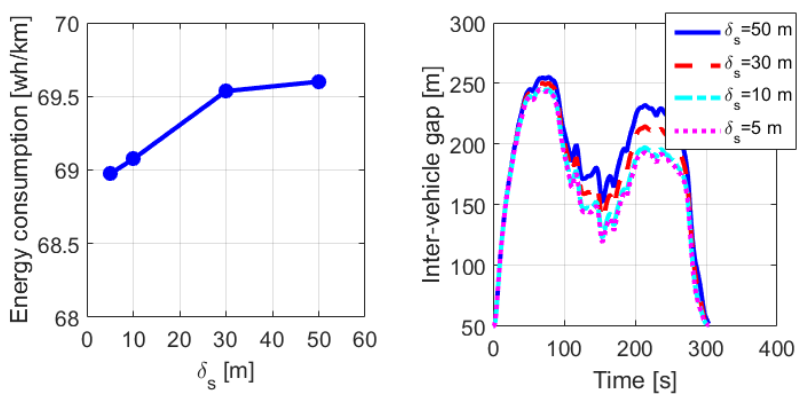

Fig. 7. Effect of the the minimum safe distance on energy consumption and inter-vehicle gap trajectory for the second driving scenario

3) Summary: As for the robustness, Fig. 8 shows the terminal time and position over time for the second driving scenario of Fig. 4. According to the predicted driving behavior of the preceding vehicle, if set terminal condition $\left(t_{p . s}, S_{s}\right)$ is feasible, it is held; otherwise it is adjusted. It is shown that terminal position (non-stop scenario) or both of terminal time and position (stop-scenario) are adjusted.
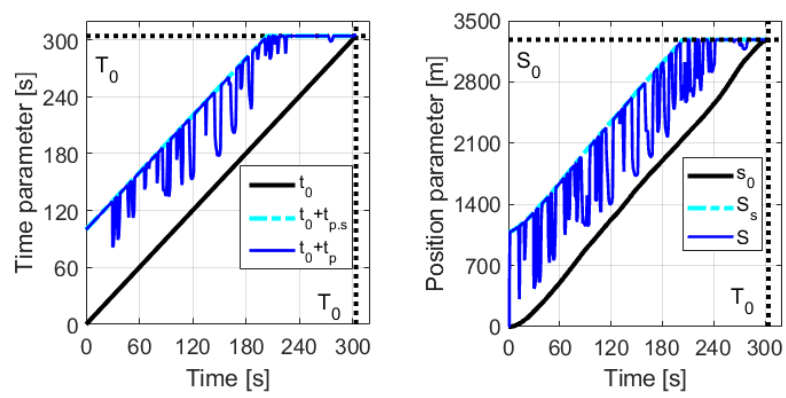

Fig. 8. Results of terminal position condition adjustment with optimized initial prediction horizon $\left(T_{p}=T_{p}^{*}=100 \mathrm{~s}\right)$ under the second scenario. $t_{0}$ and $s_{0}$ are initial time and position, respectively; $t_{0}+t_{p}$ and $S$ are final terminal time and position, respectievely; $t_{0}+t_{p . s}$ and $S_{s}$ are set terminal time and position, respectievely.

As shown in Table II, use of the optimized prediction horizon results in small loss of energy optimality (less than $8 \%$ ) and outperforms the preceding vehicle by more than 8 $\%$ (44\% at most) for all driving scenarios.

TABLE II

SUMMARY OF RESULTS FOR FOUR DRIVING SCENARIOS

\begin{tabular}{c||c|ccc}
\hline & & Preceding vehicle & MPC & Reference \\
\hline \hline Final energy & $\# 1$ & 91.3 & 65.0 & 61.9 \\
consumption & $\# 2$ & 94.0 & 69.0 & 65.8 \\
{$[w h / k m]$} & $\# 3$ & 91.1 & 86.6 & 82.6 \\
& $\# 4$ & 151.2 & 140.4 & 129.6 \\
\hline \hline Loss of & $\# 1$ & 51.6 & 7.63 & 0 \\
energy optimality & $\# 2$ & 45.1 & 6.32 & 0 \\
{$[\%]$} & $\# 3$ & 18.7 & 5.77 & 0 \\
& $\# 4$ & 16.7 & 8.33 & 0 \\
\hline
\end{tabular}




\section{CONCLUSION}

This paper presents a novel safe- and eco-driving control system based on analytical solution of energy-minimal torque input for electric CAVs. Vehicle safety is considered as a state constraint, and analytical state-constrained solutions that minimize energy consumption are derived under some assumptions. Furthermore, the feasible range of the terminal conditions is analyzed to ensure the existence of the analytical solution for all possible preceding vehicle scenarios.

The effectiveness of the proposed system is validated for several scenarios in which the preceding vehicle travels with a real-world speed profile. The simulation results show that the proposed system allows the electric CAV to accelerate/decelerate optimally, and thus increases energy efficiency without increasing the trip time, while avoiding rear-end collisions as well as maintaining a speed lower than the maximum speed limit. In addition, it is shown that the proposed system is suitable for real-time use, thanks to its low computational time. Future work is expected to include investigation of CAV's influence on mixed traffic with different penetration rates, as well as extension to a multi-lane driving scenario.

\section{APPENDIX A}

\section{COEFFICIENTS FOR STATE-CONSTRAINED SOLUTION}

\section{A. Coefficients for Speed Only Constrained Case}

$$
\begin{aligned}
A_{1.1}= & v_{0}\left(3 v_{\text {max }}^{2}-3 v_{0} v_{\max }+v_{0}^{2}\right), \\
A_{1.2}= & -6\left(S v_{0}^{2}+S v_{\max }^{2}-t_{p} v_{\max }^{3}\right. \\
& \left.+2 t_{p} v_{0} v_{\text {max }}^{2}-t_{p} v_{0}^{2} v_{\max }-2 S v_{0} v_{\max }\right), \\
A_{1.3}= & -9\left(t_{p}^{2} v_{\text {max }}^{3}-S^{2} v_{0}+S^{2} v_{\max }\right. \\
& \left.-2 S t_{p} v_{\text {max }}^{2}-t_{p}^{2} v_{0} v_{\max }^{2}+2 S t_{p} v_{0} v_{\max }\right), \\
B_{1.1}= & v_{0}-v_{\max }, \\
B_{1.2}= & 3\left(s_{0}-S\right)+t_{p} V+2 t_{p} v_{\max }, \\
B_{1.3}= & V-v_{\max }, \\
C_{1.1}= & -\left(b_{1} c_{0}\right) / c_{1}, \\
C_{1.2}= & 4 b_{2}\left(v_{0}-v_{\max }\right) / c_{1}^{2}, \\
D_{1.1}= & -\left(2 b_{2} c_{0}+b_{1} c_{1} v_{0}\right) / c_{1}^{2}, \\
D_{1.2}= & 4 b_{2}\left(v_{0}-v_{\max }\right) / c_{1}^{2} .
\end{aligned}
$$

\section{B. Coefficients for Position Only Constrained Case}

\section{1) Boundary Interval:}

$$
\begin{aligned}
& A_{2 . b .1}=-3\left(s_{0}-s_{p .0}\right), \\
& A_{2 . b .2}=v_{0}-v_{p .0} \\
& B_{2 . b .1}=-\left(3 s_{p .0}-3 S+t_{p} V+2 t_{p} v_{p .0}+\left(a_{p .0} t_{p}^{2}\right) / 2\right), \\
& B_{2 . b .2}=v_{p .0}-V+a_{p .0} t_{p}, \\
& C_{2 . b .1}=-\left(b_{1} c_{0}\right) / c_{1}, \\
& C_{2 . b .2}=4 b_{2}\left(v_{0}-v_{p .0}\right) / c_{1}^{2} \\
& D_{2 . b .1}=-\left(2 a_{p .0} b_{2}+2 b_{2} c_{0}+b_{1} c_{1} v_{0}\right) / c_{1}^{2}, \\
& D_{2 . b .2}=4 b_{2}\left(v_{0}-v_{p .0}\right) / c_{1}^{2}, \\
& E_{2 . b .1}=4 b_{2}\left(v_{p .0}-V+a_{p .0} t_{p}\right) / c_{1}^{2}, \\
& E_{2 . b .2}=4 b_{2}\left(v_{0}-v_{p .0}\right) / c_{1}^{2},
\end{aligned}
$$

$$
\begin{aligned}
& E_{2 . b .3}=-8 b_{2} t_{p}\left(v_{0}-v_{p .0}\right) / c_{1}^{2}, \\
& E_{2 . b .4}=4 b_{2} t_{p}^{2}\left(v_{0}-v_{p .0}\right) / c_{1}^{2} .
\end{aligned}
$$

\section{2) Contact Point:}

$$
\begin{aligned}
& A_{2 . c .1}=v_{0}-V+a_{p .0} t_{p}, \\
& A_{2 . c .2}=3 s_{0}-3 S-2 t_{p} v_{0}+t_{p} V+4 t_{p} v_{p .0}+\left(a_{p .0} t_{p}^{2}\right) / 2, \\
& A_{2 . c .3}=-6 t_{p}\left(s_{0}-s_{p .0}\right)+t_{p}^{2}\left(v_{0}-v_{p .0}\right), \\
& A_{2 . c .4}=3 t_{p}^{2}\left(s_{0}-s_{p .0}\right), \\
& C_{2 . c .1}=-\left(b_{1} c_{0}\right) / c_{1}, \\
& C_{2 . c .2}=12 b_{2}\left(v_{0}-v_{p .0}\right) / c_{1}^{2}, \\
& C_{2 . c .3}=24 b_{2}\left(s_{0}-s_{p .0}\right) / c_{1}^{2}, \\
& D_{2 . c .1}=-\left(2 a_{p .0} b_{2}+2 b_{2} c_{0}+b_{1} c_{1} v_{0}\right) / c_{1}^{2}, \\
& D_{2 . c .2}=8 b_{2}\left(v_{0}-v_{p .0}\right) / c_{1}^{2}, \\
& D_{2 . c .3}=12 b_{2}\left(s_{0}-s_{p .0}\right) / c_{1}^{2}, \\
& E_{2 . c .1}=4 b_{2}\left(v_{0}-V+a_{p .0} t_{p}\right) / c_{1}^{2}, \\
& E_{2 . c .2}=16 b_{2} t_{p}\left(v_{0}-v_{p .0}\right) / c_{1}^{2}, \\
& E_{2 . c .3}=12 b_{2} t_{p}\left(2\left(s_{0}-s_{p .0}\right)-t_{p}\left(v_{0}-v_{p .0}\right)\right) / c_{1}^{2}, \\
& E_{2 . c .4}=24 b_{2} t_{p}^{2}\left(s_{0}-s_{p .0}\right) / c_{1}^{2} .
\end{aligned}
$$

\section{REFERENCES}

[1] L. Bertoni, J. Guanetti, M. Basso, M. Masoero, S. Cetinkunt, and F. Borrelli, "An adaptive cruise control for connected energy-saving electric vehicles," in Proc. 20th World Congress IFAC, Toulouse, France, 2017, pp. 2395-2400.

[2] K. Yu, J. Yang, and D. Yamaguchi, "Model predictive control for hybrid vehicle ecological driving using traffic signal and road slope information," Control Theory and Technology, vol. 13, pp. 17-28, Feb. 2015.

[3] M. A. S. Kamal and T. Kawabe, "Eco-driving using real-time optimization," in Proc. 2015 European Control Conference (ECC), Linz, Austria, 2015, pp. 111-116.

[4] D. Lang, T. Stanger, R. Schmied, and L. del Re, "Predictive cooperative adaptive cruise control: Fuel consumption benefits and implementability," in Optimization and Optimal Control in Automotive Systems, ser. Lecture Notes in Control and Information Sciences, H. Waschl, I. Kolmanovsky, M. Steinbuch, and L. del Re, Eds. Springer International Publishing, 2014.

[5] M. Vajedi and N. L. Azad, "Ecological adaptive cruise control for plugin hybrid electric vehicles using nonlinear model predictive control," IEEE Transactions on Intelligent Transportation Systems, vol. 17, no. 1, pp. 113-122, 2016.

[6] T. Ohtsuka, "A continuation/gmres method for fast computation of nonlinear receding horizon control," Automatica, vol. 40, pp. 563-574, Apr. 2004.

[7] M. Huang, H. Nakada, K. Butts, and I. Kolmanovsky, "Nonlinear model predictive control of a diesel engine air path: A comparison of constraint handling and computational strategies," in Proc. 5th IFAC Conference on Nonlinear Model Predictive Control NMPC 2015, vol. 48, no. 23 , Seville, Spain, Sep. 2015, pp. 372-379.

[8] S. Tajeddin, M. Vajedi, and N. L. Azad, "A newton/gmres approach to predictive ecological adaptive cruise control of a plug-in hybrid electric vehicle in car-following scenarios," in Proc. 7th IFAC Symposium on Mechatronic Systems MECHATRONICS 2016, vol. 49, no. 21, Leicestershire, UK, Sep. 2016, pp. 59-65.

[9] M. A. S. Kamal, M. Mukai, J. Murata, and T. Kawabe, "Model predictive control of vehicles on urban roads for improved fuel economy," IEEE Transactions on Control Systems Technology, vol. 21, pp. 831-841, May 2013.

[10] A. Sciarretta, G. De Nunzio, and L. L. Ojeda, "Optimal ecodriving control: Energy-efficient driving of road vehicles as an optimal control problem," IEEE Control Systems, vol. 35, no. 5, pp. 71-90, Oct. 2015.

[11] E. Ozatay, S. Onori, J. Wollaeger, U. Ozguner, G. Rizzoni, D. Filev, J. Michelini, and S. D. Cairano, "Cloud-based velocity profile optimization for everyday driving: A dynamic-programming-based solution," IEEE Transactions on Intelligent Transportation Systems, vol. 15, no. 6 , pp. 2491-2505, Dec. 2014. 
[12] F. Mensing, E. Bideaux, R. Trigui, and H. Tattegrain, "Trajectory optimization of electric vehicles for eco-driving applications," Transportation Research Part D: Transport and Environment, vol. 18, 12013.

[13] G. Mahler and A. Vahidi, "An optimal velocity-planning scheme for vehicle energy efficiency through probabilistic prediction of trafficsignal timing," IEEE Transactions on Intelligent Transportation Systems, vol. 15, no. 6, pp. 2516-2523, Dec. 2014

[14] W. Dib, A. Chasse, P. Moulin, A. Sciarretta, and G. Corde, "Optimal energy management for an electric vehicle in eco-driving applications," Control Engineering Practice, vol. 29, pp. 299-307, Aug. 2014.

[15] N. Wan, A. Vahidi, and A. Luckow, "Optimal speed advisory for connected vehicles in arterial roads and the impact on mixed traffic," Transportation Research Part C: Emerging Technologies, vol. 69, pp. 548-563, Aug. 2016.

[16] E. Ozatay, U. Ozguner, and D. Filev, "Velocity profile optimization of on road vehicles: Pontryagin's maximum principle based approach," Control Engineering Practice, vol. 51, Oct. 2016.

[17] J. Rios-Torres and A. A. Malikopoulos, "Automated and cooperative vehicle merging at highway on-ramps," IEEE Transactions on Intelligent Transportation Systems, vol. PP, no. 99, pp. 1-10, Aug. 2016.

[18] Y. J. Zhang, A. A. Malikopoulos, and C. G. Cassandras, "Optimal control and coordination of connected and automated vehicles at urban traffic intersections," in Proc. 2016 American Control Conference (ACC), Boston, MA, 2016, pp. 6227-6232.

[19] Z. Yue, C. G. Cassandras, and A. A. Malikopoulos, "Optimal control of connected automated vehicles at urban traffic intersections: A feasibility enforcement analysis," will present at the 2017 American Control Conference (ACC), Seattle, WA, May 2017.

[20] L. Guzzella and A. Sciarretta, Vehicle Propulsion Systemsl: Introduction to Modeling and Optimization. Springer-Verlag Berlin Heidelberg, 2013.

[21] J. M. Maestre, D. Muoz de la Pea, and E. F. Camacho, "Distributed model predictive control based on a cooperative game," Optimal Control Applications and Methods, vol. 32, no. 2, pp. 153-176, 2011.

[22] A. Ferrara, A. N. Oleari, S. Sacone, and S. Siri, "An event-triggered model predictive control scheme for freeway systems," in 2012 IEEE 51st IEEE Conference on Decision and Control (CDC), Dec. 2012, pp. 6975-6982.

[23] N. Petit and A. Sciarretta, "Optimal drive of electric vehicles using an inversion-based trajectory generation approach," in Proc. the 18th IFAC World Congress, Millano, Italy, Aug. 2011, pp. 14 519-14526.

[24] R. F. Hartl, S. P. Sethi, and R. G. Vickson, "A survey of the maximum principles for optimal control problems with state constraints," SIAM Review, vol. 37, pp. 181-218, 1995.

[25] A. E. Bryson and Y.-C. Ho, Applied optimal control: optimization, estimation, and control. Washington, DC: CRC Press, 1975.

[26] A. Sciarretta, L. Serrao, P. Dewangan, P. Tona, E. Bergshoeff, C. Bordons, L. Charmpa, P. Elbert, L. Eriksson, T. Hofman, M. Hubacher, P. Isenegger, F. Lacandia, A. Laveau, H. Li, D. Marcos, T. Nesch, S. Onori, P. Pisu, J. Rios, E. Silvas, M. Sivertsson, L. Tribioli, A.J. van der Hoeven, and $\mathrm{M}$. Wu, "A control benchmark on the energy management of a plug-in hybrid electric vehicle," Control Engineering Practice, vol. 29, pp. 287-298, 2014.

[27] P. Malisanil, F. Chaplais, and N. Petit, "An interior penalty method for optimal control problems with state and input constraints of nonlinear systems," Optimal Control Applications and Methods, vol. 37, pp. 3-33, 2016.

[28] L. Shampine, M. Reichelt, and J. Kierzenka. (2000) Solving boundary value problems for ordinary differential equations in matlab with bvp4c. Tutorial. [Online]. Available: http://www.mathworks.com/

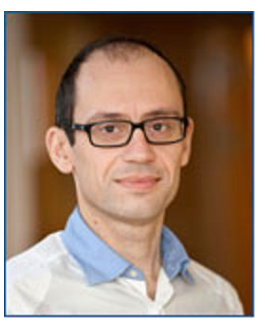

Antonio Sciarretta is an expert researcher with IFP Energies Nouvelles, Rueil Malmaison, France, since 2006. He received a M.Sc. (Mech. Eng.) degree in 1995, and a Doctorate degree (Thermal machines) in 1999, both from the University of LAquila, Italy. He earned a Habilitation (Control Eng.) in 2009 from the Grenoble Institute of Technology, France. Before joining IFPEN he has hold various teaching and research positions at the University of LAquila and at the ETH Zurich. From 2009 to 2016 he has hold the Tuck foundation Chair on Hybrid vehicles and energy management at IFP School. He has actively contributed to the development of hybrid-vehicle activities at IFPEN and he is currently managing research projects on the control and optimization for connected and automated vehicles. He has coauthored the Springer book Vehicle Propulsion Systems (three editions) and contributes to the scientific and professional community as a journal associate editor, conference organizer, and project evaluator.

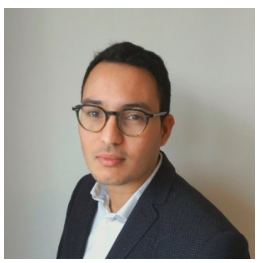

Luis Leon Ojeda received the B.Sc. degree in Electrical Engineering from the Universidad Central de Venezuela, In 2006. He received his M.S. degree in Systems Engineering from the University La Sapienza, Roma, Italy, in 2008, and the Ph.D. in Automatic Control from the University of Grenoble, France, in July 2014. He is currently a research engineer at the IFP Energies Nouvelles, Paris. His research interests are in the area of intelligent transportation systems, specifically, traffic estimation, forecasting, data treatment, traffic simulation, vehicle powertrain modeling, advanced driver-assistance systems (ADAS), and optimization of energy efficiency.

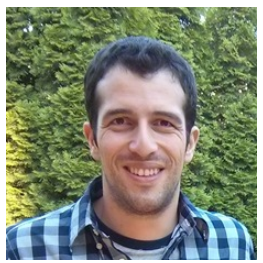

Giovanni De Nunzio received the B.Sc. and M.Sc. degrees in information and automation engineering at the University of LAquila, Italy, in 2007 and 2010. He received the doctorate degree in automatic control from the Grenoble Institute of Technology, France, in 2015. He was a visiting researcher at the Mechanical Engineering department at U.C. Berkeley in 2014. He is currently a research engineer at IFP Energies nouvelles, Lyon, France. His research activities focus on modeling, control, and simulation of traffic and transportation systems, vehicle powertrain modeling, advanced driver-assistance systems, energy consumption minimization, graph theory.

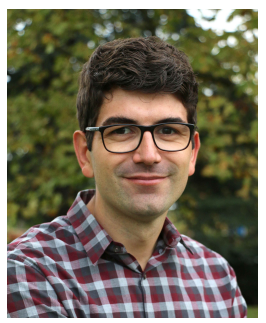

Laurent Thibault received the M.S. degrees in information and automation engineering at Supelec, France, in 2010 and in powertrain design at IFP School, in 2011. He is currently a research project leader at IFP Energies nouvelles, Lyon, France. His research activities focus on modeling, control, and simulation of connected vehicle and pollutant emissions.

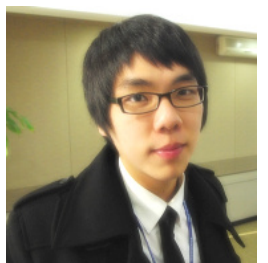

Jihun Han received his B.Sc., M.Sc. and Ph.D degrees in Mechanical Engineering from Korea Advanced Institute of Science and Technology (KAIST), South Korea, in 2009, 2011 and 2016, respectively. He was a postdoctoral research associate with IFP Energies Nouvelles, Rueil Malmaison, France in 2016-2017. He is currently a postdoctoral research associate with Oak Ridge National Laboratory, Oak Ridge, USA. His research focuses on optimal control and simulation with an emphasis in 